\title{
Posteromedial Approach to Fix Posterior Cruciate Ligament Tibial Avulsion: A Backup Fixation and Case
}

\section{Series}

\author{
Elhadi Babikir, MBBS, CABMS ${ }^{*}$ (D), Mohd AlKhayarin, FRCSC, FRCSI', Waleed \\ Asad, $M D^{1}$ and Ahmed Khalil Attia, $M D^{3}$ \\ ${ }^{1}$ Orthopedic Trauma Fellow Hamad General Hospital, Doha, Qatar \\ ${ }^{2}$ Assistant Professor of Clinical Orthopedic Surgery, Weil Cornel University Qatar, Senior Consultant at Hamad General \\ Hospital, Qatar \\ ${ }^{3}$ Orthopedics Senior Resident at Hamad General Hospital, Doha, Qatar
}

\begin{abstract}
Background and purpose: Tibial avulsion fractures of PCL are common; however, the choice between open reduction internal fixation (ORIF) and arthroscopic repair of acute fractures remains controversial. We assessed the efficacy and safety of managing PCL avulsion fractures with ORIF using the posteromedial approach.

Methods: This series includes 10 patients (9 males and one female) with a median age of 36 years (range 23-48 years) who underwent ORIF with a posteromedial approach for PCL avulsion fractures, with and without other knee ligamentous injuries. Bony fragments were fixed using cortical screw with a washer augmented with another backup screw fixed 3-5 $\mathrm{cm}$ distally. Postoperatively, the mean follow-up time was 18.8 months (range 3.5-48 months). All the surgeries were performed at a mean of 75 days (range 1-600 days) of injury, and clinical and radiological assessments were also used.

Results: The patients achieved fracture union an average of 14 weeks postoperatively (range: 8-24 weeks). Two patients who sustained other ligamentous injuries demonstrated residual disability. Patients returned to work (heavy labour) within 6 months postoperatively (range: 4-9 months).

Conclusion: The posteromedial approach is safe, time saving, and was not associated with complications. This approach provides stable fixation with no residual knee laxity and can be used to manage posteromedial fractures of the medial femoral condyle and tibial plateau. Further high quality studies are needed to assess this surgical approach.
\end{abstract}

\section{Keywords}

Fractures, Open reduction internal fixation, Posterior cruciate ligament tibial avulsion, Posteromedial approach, PCL, Backup fixation

\section{Abbreviations}

RTA: Road Traffic Accident; FFH: Fall From Height; M: Male; F: Female; R: Right; L: Left; Bil: Bilateral; MCL: Medial Collateral; LCL: Lateral Collateral; PCL: Posterior Cruciate Ligament; PLC: Posterolateral Corner; ROM: Range of Motion

\section{Introduction}

Tibial avulsion fractures of the Posterior cruciate ligament $(\mathrm{PCL})$ are common; however, methods of surgical treatment are insufficiently described. $\mathrm{PCL}$ is the main posterior stabilizer of the knee and limits posterior tibial translation relative to the femur [1,2]. In addition, the PCL serves as a central axis controlling and imparting rotational stability to the knee. Isolated tear or avulsion of the PCL can be caused by falling onto a flexed knee or by striking the flexed tibia on the dashboard
*Corresponding author: Dr. Elhadi Babikir, MBBS, CABMS, Orthopedic Trauma Surgeon, Hamad General Hospital, Doha, 3050, Qatar, Tel: +974-4439-2820; +974-4439-2823

Accepted: June 08, 2021

Published online: June 10, 2021

Citation: Babikir E, AlKhayarin M, Asad W, et al. (2021) Posteromedial Approach to Fix Posterior Cruciate Ligament Tibial Avulsion: A Backup Fixation and Case Series. J Orthop Surg Tech $4(1): 319-324$ 
Citation: Babikir E, AlKhayarin M, Asad W, et al. (2021) Posteromedial Approach to Fix Posterior Cruciate Ligament Tibial Avulsion: A Backup Fixation and Case Series. J Orthop Surg Tech 4(1):319-324

during a motor vehicle accident [3]. Isolated PCL disruption most commonly occurs as avulsion at the point of tibial insertion, as opposed to its femoral origin or as a mid-substance tear. The tibial insertion of the PCL is also more consistent than the femoral insertion [4]. The two PCL fibre bundles insert, without anatomic separation, into the centrally-located fovea or facet on the posterior aspect of the tibia, 1-1.5 cm distal to the joint line, with the posterior horn of the medial meniscus being the anterior-most extension [5]. Chronic disability and early degenerative changes can develop as a result of delayed diagnosis.

The posteromedial knee approach is a surgical technique for treating tibial avulsion fractures of the PCL. The technique involves reinsertion and fixation of the PCL bone fragment into its anatomical bed located on the posterior tibial surface. The posteromedial knee approach is reportedly sufficient for re-establishing ligament integrity and function; however, most surgeons are not familiar with this surgical approach because it is poorly-described within the published literature.
Backup fixation is not known to the orthopedic community.

\section{Methods}

This is a case series of 10 patients that presented to our hospital from 2007 to 2020 . Patients included in the study ( 9 males, 1 female) had a median age of 36 years (range 24-48 years), and had all sustained PCL avulsion fractures of various aetiologies [ 7 patients by road traffic accident (RTA), 3 patients by a fall from height (FFH)]. Four patients sustained other knee ligamentous injuries while in 6 patients the injury was isolated (Table 1). All the patients underwent open reduction internal fixation (ORIF) of PCL avulsion fractures, from the tibia, using the posteromedial approach at a mean of 75 days (range 1-600 days) from the injury. Surgical delays were mainly due to non-orthopaedic injuries (e.g., chest or abdominal injuries which necessitatedprolonged intensive care). The same experienced surgeon (senior author) performed all the surgeries at a level 1 trauma center.

Each patient underwent standard preoperative assess-

Tables 1: Patients with knee ligamentous injuries and clinical outcome.

\begin{tabular}{|c|c|c|c|c|c|c|c|c|c|}
\hline No. & $\begin{array}{l}\text { Agel } \\
\text { Sex }\end{array}$ & Side & $\begin{array}{l}\text { Associated } \\
\text { injury }\end{array}$ & $\begin{array}{l}\text { Time between } \\
\text { injury \& surgery }\end{array}$ & $\begin{array}{l}\text { Time to } \\
\text { Union }\end{array}$ & ROM & $\begin{array}{l}\text { Follow } \\
\text { up }\end{array}$ & Instability & $\begin{array}{l}\text { Return to } \\
\text { work }\end{array}$ \\
\hline 1. & $27 / F$ & $\mathrm{~L}$ & LCL, PLC & 20 months & 14 weeks & $-5-105$ & $48 m$ & Unstable & 9 months \\
\hline 2. & $45 / \mathrm{M}$ & Bil & Right: PLC & $\begin{array}{l}\text { Rt } 18 \text { days, Lt } 6 \\
\text { days }\end{array}$ & $\begin{array}{l}\text { R: } 18 \text { weeks } \\
\text { L: } 12 \text { weeks }\end{array}$ & $\begin{array}{l}\text { R: } 2-118 \\
\text { L: } 2-115\end{array}$ & $12 m$ & $\begin{array}{l}\text { Right } \\
\text { unstable }\end{array}$ & - \\
\hline 3. & 29/M & $L$ & None & 8 days & 8 weeks & $0-142$ & $11 \mathrm{~m}$ & Nil & 4 months \\
\hline 4. & $43 / \mathrm{M}$ & $\mathrm{R}$ & None & 17 days & 12 weeks & $0-140$ & $9 m$ & Nil & 9 months \\
\hline 5. & $24 / \mathrm{M}$ & $\mathrm{R}$ & None & 23 days & 12 weeks & $4-142$ & $36 \mathrm{~m}$ & Nil & 6 months \\
\hline 6. & $45 / \mathrm{M}$ & $L$ & None & 63 days & 12 weeks & $0-140$ & $16 \mathrm{~m}$ & Nil & 6 months \\
\hline 7. & $48 / \mathrm{M}$ & $R$ & $\mathrm{MCL}$ & 9 days & 24 weeks & $0-140$ & $33 m$ & Nil & 8 months \\
\hline 8. & 29/M & $R$ & None & 1 day & 10 weeks & $0-140$ & $9 m$ & Nil & 6 months \\
\hline 9. & $26 / \mathrm{M}$ & $L$ & None & 10 days & 10 weeks & $0-140$ & $11 \mathrm{~m}$ & Nil & 11 months \\
\hline 10. & $23 / \mathrm{M}$ & $L$ & MCL & 10 days & 14 weeks & $0-140$ & $3.5 \mathrm{~m}$ & Nil & - \\
\hline
\end{tabular}

M: Male; F: Female; R: Right; L: Left; Bil: Bilateral; MCL: Medial Collateral; LCL: Lateral Collateral; PCL: Posterior Cruciate Ligament; PLC: Posterolateral Corner; ROM: Range of Motion.
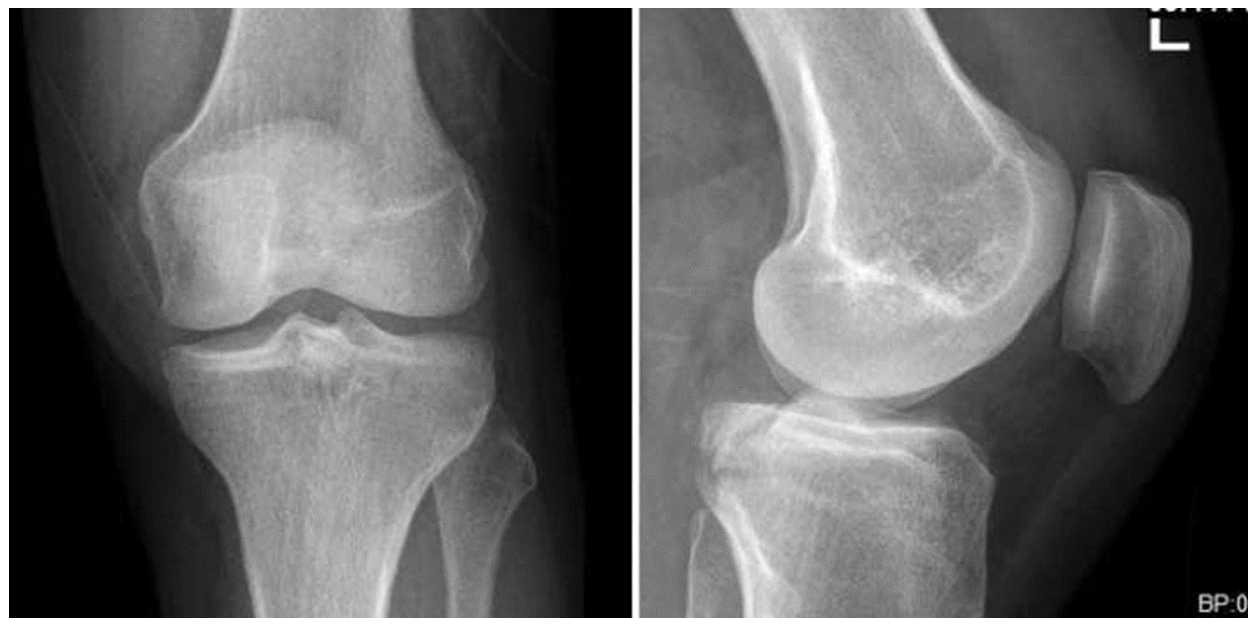

Figure 1: Preoperative AP and Lateral X-rays. 
Citation: Babikir E, AlKhayarin M, Asad W, et al. (2021) Posteromedial Approach to Fix Posterior Cruciate Ligament Tibial Avulsion: A Backup Fixation and Case Series. J Orthop Surg Tech 4(1):319-324

ment to better define knee instability and other associated injuries. The diagnosis was confirmed using AP and lateral radiographs of the knee (Figure 1), followed by MRI in 3 patients to rule out occult mid-substance PCL injuries and other intraarticular pathologies or associated ligamentous injuries (Figure 2). Three patients received a CT scan to further delineate a bony injury (Figure 3). All data were obtained from patients' charts and outpatient records following final approval from the institutional review board (IRB). Bony fragments were fixed using cortical screw with a washer augmented with another backup screw fixed 3-5 cm distally.

\section{Operative Technique}

A tourniquet was applied high up in the thigh. Then, the patients underwent diagnostic arthroscopy of the knee while in the supine position. Following that, the patient was flipped into the prone position and re-prepped. The lower extremity was held at 30 degrees of flexion at the knee joint by placing a bolster at the ankle. We followed a simplified approach, as described by Burks and Schaffer [6], consisting of an inverted L-incision made over the posteromedial corner of the knee joint. The skin incision consisted of a gentle curve with the
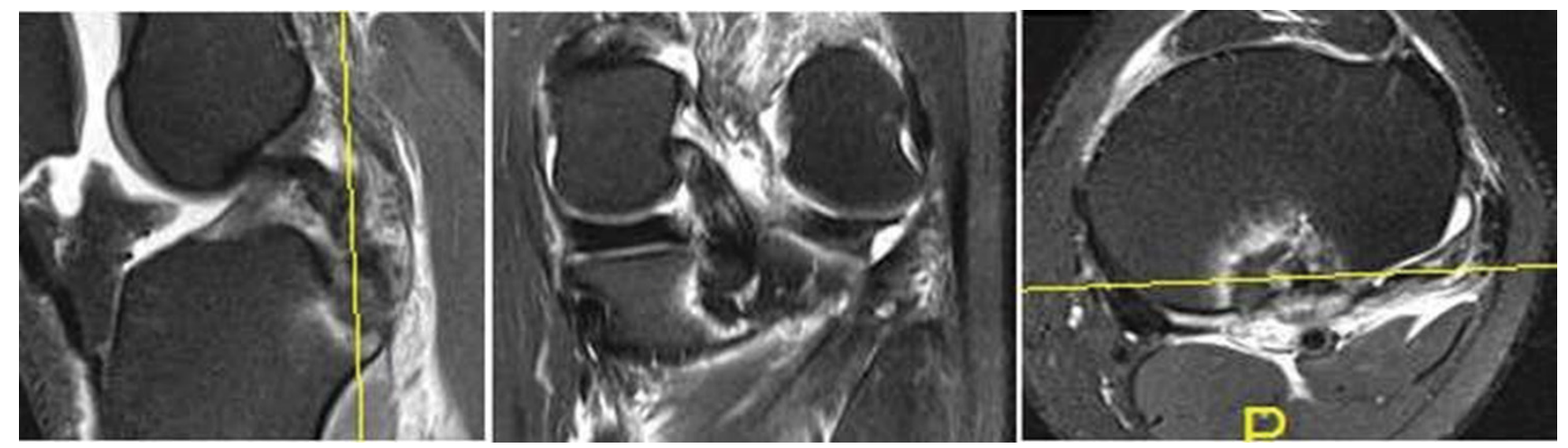

Figure 2: Preoperative MRI (sagittal, coronal, and axial, respectively).
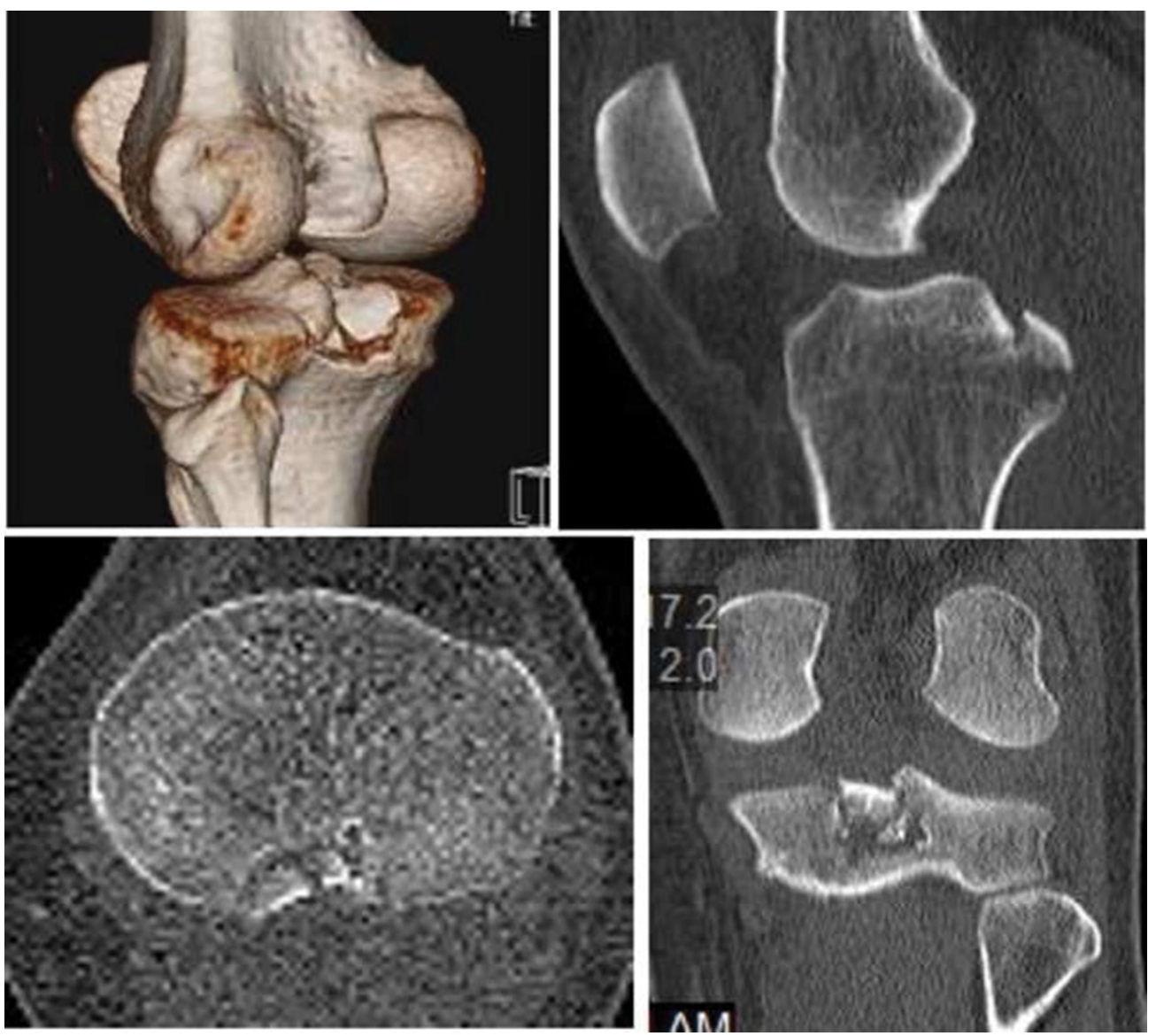

Figure 3: Preoperative CT scan (3D, Axial, sagittal, and coronal, respectively). 


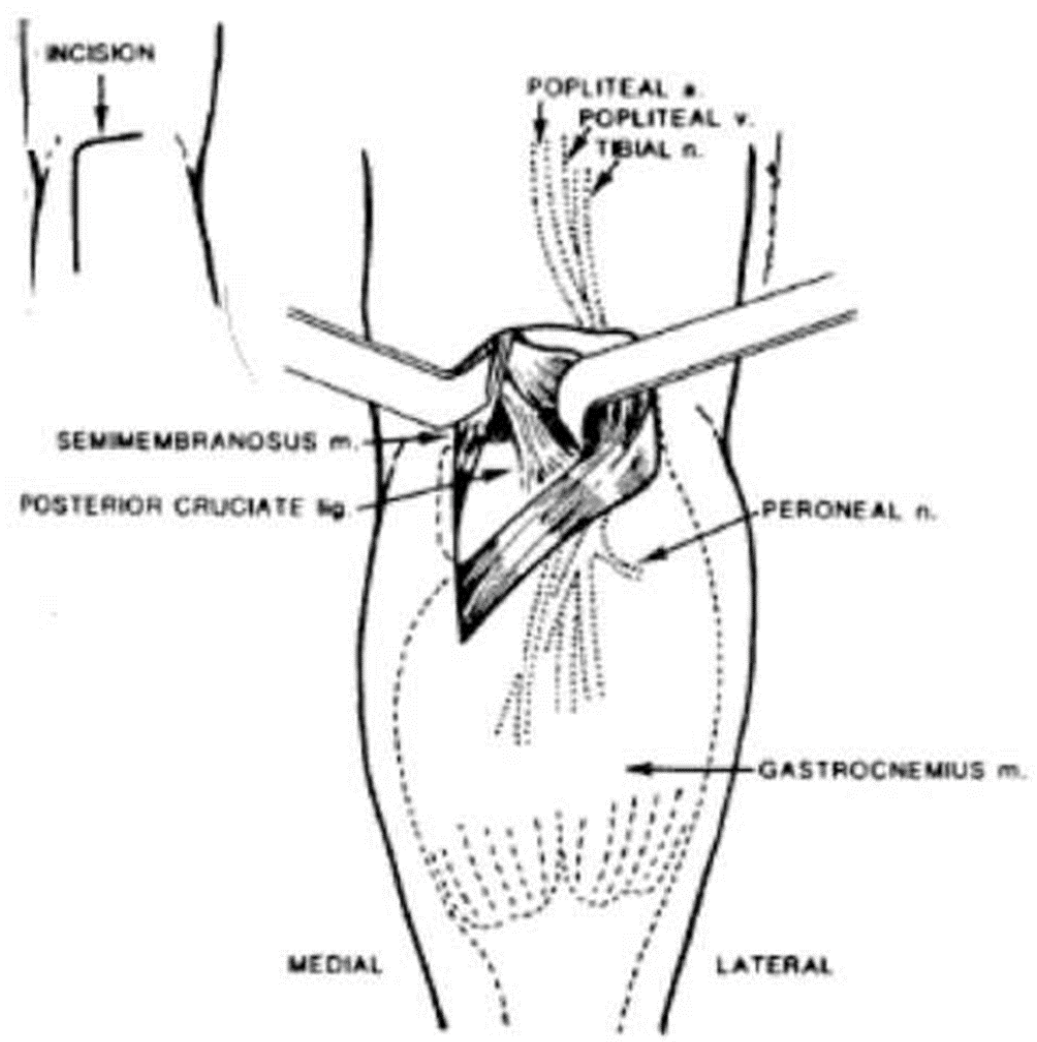

Figure 4: This figure was reproduced with permission [6].
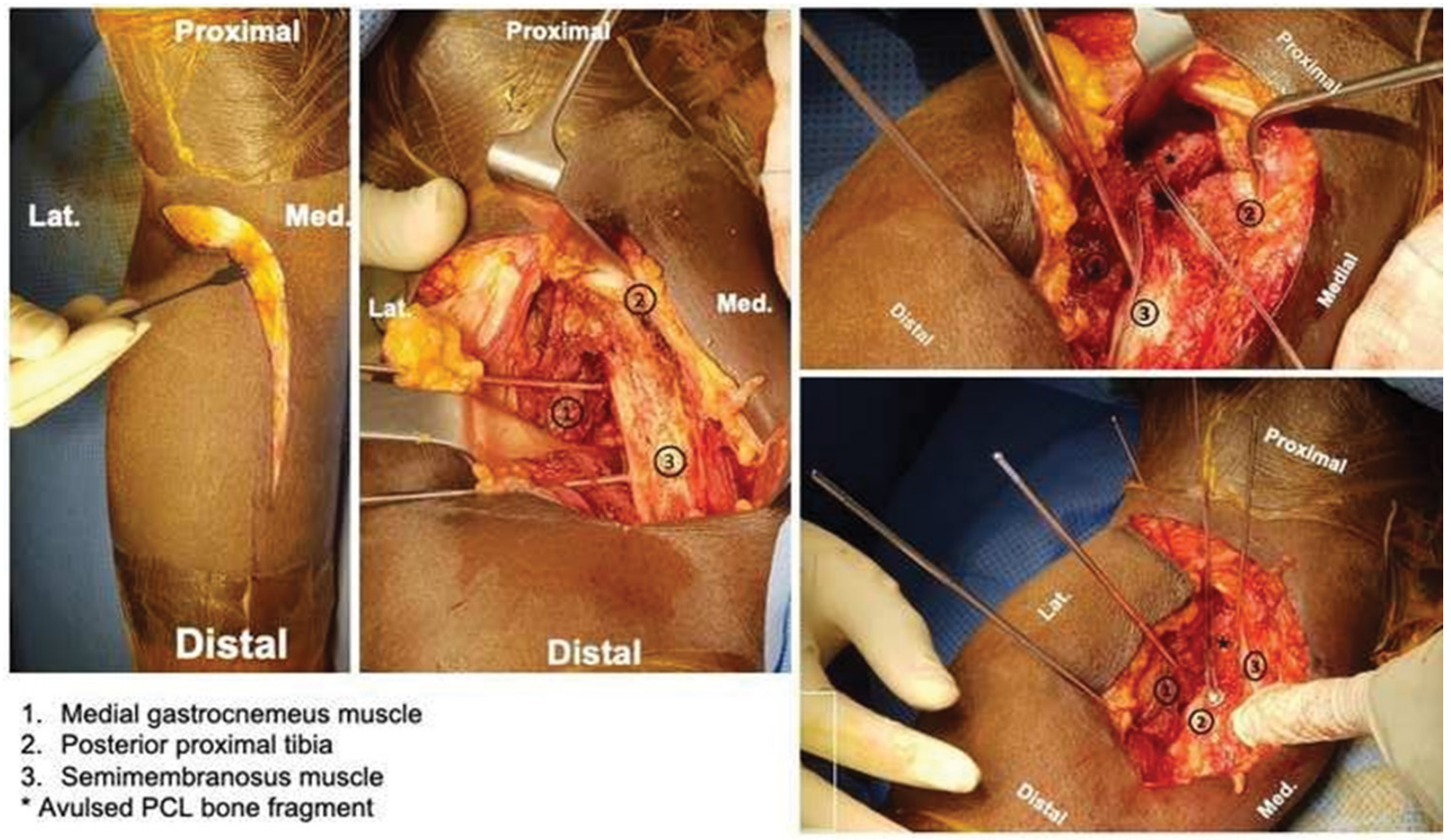

1. Medial gastrocnemeus muscle

2. Posterior proximal tibia

3. Semimembranosus muscle

* Avulsed PCL bone fragment

Figure 5: Clinical intraoperative photograph of surgical approach. 
horizontal end at the flexion crease of the knee, and a vertical limb overlying the medial aspect of the medial gastrocnemius muscle. Dissection was carried to the deep fascial layer, which was incised vertically over the medial head of the gastrocnemius. The medial border of the "medial" gastrocnemius was identified, and we then developed the interval between it and the semimembranosus tendon. This plane was developed by blunt dissection until we reached the posterior joint capsule. The medial head of gastrocnemius was retracted "laterally" so that neurovascular structures were kept away from the surgical field (Figure 4 and Figure 5).

At this point, a vertical incision was made through the posterior capsule. The contents of the posterior intercondylar notch and the tibial attachment of the PCL were exposed. The bony fragment was pushed down to its bed which was freshened and prepared, and then secured with provisional $\mathrm{K}$-wire fixation. The fluoroscope was used to verify the appropriate position. Two Krakow type sutures, usually consisting of non-absorbable suture, were used in the tendinous part of the distal PCL. We fixed the bony fragment in its bed with a $3.5 \mathrm{~mm}$ cortical screw and a plastic-spiked ligament washer. The screw was placed at 45 degree, relative to the posterior cortex. Then, the sutures were wrapped around another 3.5 $\mathrm{mm}$ cortical screw with a regular washer fixed $3-5 \mathrm{~cm}$ distal to the first screw to serve as a backup or reinforcement fixation (like a post). The screw and sutures were tightened with the knee in a approximately 70 degrees of flexion and anterior tibial translation to restore the normal tibial step off, as shown in (Figure 6). After fixation, both the semimembranosus and medial gastrocnemius were allowed to fall back into their normal position, or were repaired if they had tendinized. The skin was closed after approximating the subcutaneous layers.

\section{Postoperative care and follow up}

All patients were kept on knee immobilizers postoperatively and carried out immediate, gentle, and full passive range of motion tasks with posterior tibial support and non weight bearing ambulation for 2 months. Isometric closed ki- netic chain exercises and patellar mobilization were immediately introduced. No active hamstring exercises were allowed for at least 12-16 weeks.

Patients were assessed clinically at 2 weeks, clinically and radiographically at 6 weeks, 3 months, 6 months and up to 36 months postoperatively. We restricted resumption of heavy work and sports until 9-12 months when sufficient strength, range of motion, and proprioceptive skills had returned.

\section{Results}

All the patients were assessed clinically by wound inspection, posterior sag, posterior drawer test, range of motion, and generalized instability. Radiological assessment was done by AP and lateral X-rays. We achieved bony union in all patients at an average of around 14 weeks (range: 8-24 weeks).

None of the patients experienced neurovascular complications, and all achieved acceptable range of motion averaging 0-130 degrees. By the last clinic visit, there were no instances of posterior sag and zero posterior drawer tests. We had 2 failures, one patient sustained posterolateral corner injury that was reconstructed and the other one was a multiply injured patient done 20 months post-injury (missed injury) and should have been reconstructed. There were no wound complications which necessitated wound debridement or hardware removal. All the patients returned to work (heavy labor) at an average of 6 months postoperatively (range: 4-9 months). Younger patients tended to have better clinical outcome and faster time to bony union (Table 1).

\section{Discussion}

Injury to the $\mathrm{PCL}$ is gaining recognition as a disabling problem that are often overlooked [7]. The choice between treatment using ORIF or arthroscopic repair of acute fractures remains controversial. Surgical fixation of the bony tibial avulsion by either a screw or k-wire is advocated and it has produced almost uniformly excellent results. Some orthopaedic surgeons are hesitant to treat tibial avulsions of the PCL be-
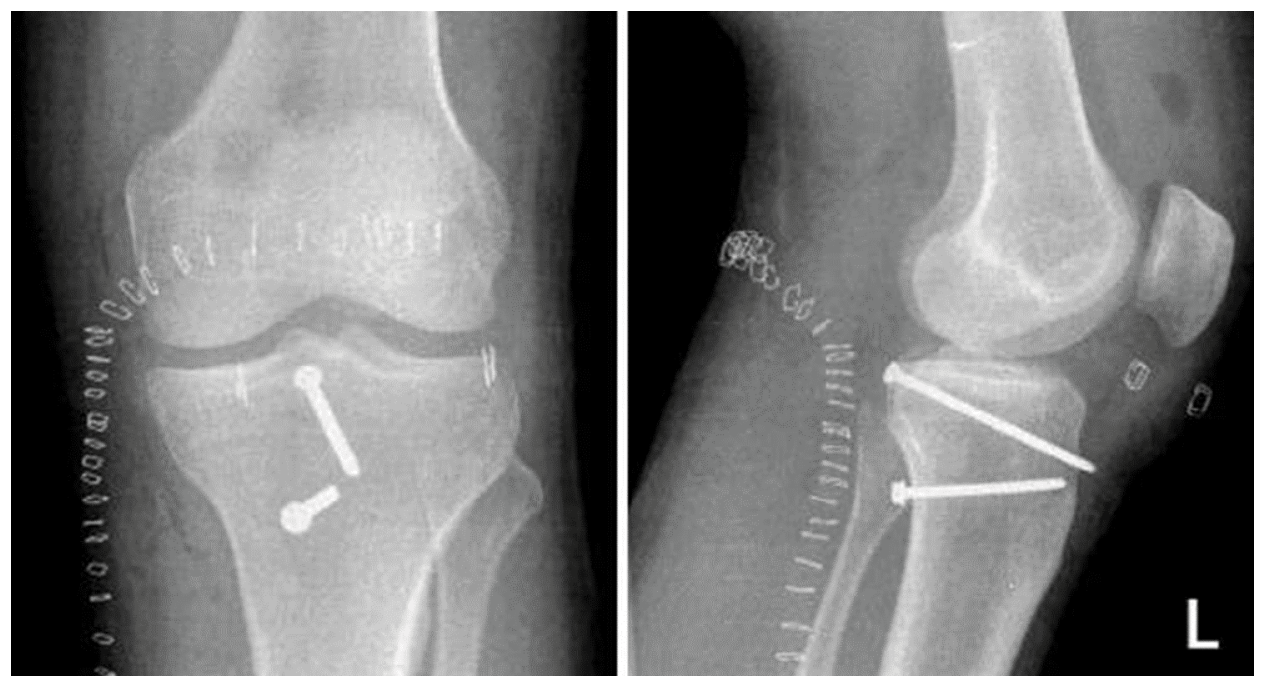

Figure 6: Postoperative AP and Lateral X-rays. 
cause of their unfamiliarity with the standard posterior approach to the knee [8]. In our study we used the modified posterior approach by Burk \& Schaffer [6] which has become the standard for approaching the PCL, either for fixing avulsions or for onlay reconstructive grafting.

Many reports showed that posteromedial approach is fairly easy, safe, time saving, and associated with less morbidity [9-12].

Frosch, et al. [13], in a small case series, advocated a minimally-invasive posteromedial approach, with the patient in the supine position, for fixing PCL avulsion fractures. Contraindications for this approach include infections in or around the knee, critical soft tissue conditions, and lack of patient compliance.

The authors sought to assess the efficacy and safety of the posteromedial approach for treatment of PCL tibial avulsion. Some limitations to this study include the small study cohort and lack of long-term follow-up which was difficult to achieve. We had 2 failures of our back up fixation, one patient sustained posterolateral corner injury that was reconstructed and the other one was a multiply injured patient done 20 months post-injury (missed injury) and should have been reconstructed.

\section{Conclusion}

The posteromedial approach to fixing PCL avulsion fractures is safe, time-saving, and associated with less morbidity. This approach assists anatomic fixation of the fragment, thereby encouraging fast union and a clinically-stable knee joint. All of the patients included in this case series achieved bone-to-bone healing without residual knee laxity or instability, with the exception of 2 patients. Chronic cases should not be fixed and should be reconstructed instead. Associated ligamentous injury have higher failure rate. More studies with larger numbers are needed.

\section{Acknowledgements}

The authors are grateful to the medical research center (Hamad Medical Corporation) for their help and support during the study period.

The authors would like to thank Enago (www.enago.com) for the English language review.

\section{Conflict of Interest}

The authors declare that they have no conflict of interest.

\section{Informed Consent}

The corresponding author collected the consent forms from all study participants for publication of this article.

\section{Funding}

There is no funding source.

\section{Ethical Approval}

IRB approval was granted by the Medical Research Center of the Hamad Medical Corporation approved this study (\#17040/17). The study was performed in compliance with Helsinki declaration.

\section{References}

1. De Abreu MR, Kim HJ, Ching CB, et al. (2005) Posterior cruciate ligament recess and normal posterior capsular insertional anatomy: MR imaging of cadaveric knees. Radiology 236: 968-973.

2. Van Dommelen BA, Flower PJ (1989) Anatomy of posterior cruciate ligament. A review. Am J Sports Med 17: 24-29.

3. Shino K, Nakata K, Mae T, et al. (2003) Arthroscopic fixation of tibial bony avulsion of the posterior cruciate ligament. Arthroscopy 19: E12.

4. Edwards A, Bull AM, Amis AA (2007) The attachments of the fiber bundles of the posterior cruciate ligament: An anatomic study. Arthroscopy 23: 284-290.

5. Dargel J, Pohl P, Tzikaras P, et al. (2006) Morphometric side-side differences in human cruciate ligament insertions. Surg Radiol Anat 28: 398-402.

6. Burks RT, Schaffer JJ (1990) A simplified approach to the tibial attachment of the posterior cruciate ligament. Clin Orthop Res 254: 216-219.

7. Kannus P, Bergfelt J, Jarvinen M, et al. (1991) Injuries to the posterior cruciate ligament of the knee. Sports Med 12: 110-131.

8. Abbott LC, Carpenter WF (1945) Surgical approaches to the knee joint. J Bone Joint Surg 27: 277-310.

9. Attia ME, Zanfaly Al (2014) Fixation of tibial bony avulsion of the posterior cruciate ligament using the posteromedial approach. The Egyptian Ortho J 49: 281-285.

10. Bali K, Prabhakar S, Saini U, et al. (2012) Open reduction and internal fixation of isolated PCL fossa avulsion fractures. Knee Surg Sports Traumatol Arthrosc 20: 315-321.

11. Lamichhane A, Mahara DP (2012) Management of posterior cruciate ligament avulsion by cannulated screw fixation. JIOM 34: 28-31.

12. Nicandri GT, Klineberg EO, Wahl CJ, et al. (2008) Treatment of posterior cruciate ligament tibial avulsion fractures through a modified open posterior approach: Operative technique and 1248 months outcomes. J Orthop Trauma 22: 317-324.

13. Frosch KH, Proksch N, Preiss A, et al. (2012) Treatment of bony avulsions of the posterior cruciate ligament $(P C L)$ by a minimally invasive dorsal approach. Operative Orthopedic Und Traumatologic 24: 348-353.

DOI: $10.36959 / 453 / 566$

Copyright: (c) 2021 Babikir E, et al. This is an open-access article distributed under the terms of the Creative Commons Attribution License, which permits unrestricted use, distribution, and reproduction in any medium, provided the original author and source are credited. 